\title{
Modeling and Simulation for Nonlinear Pressurized Water Reactor Cores Using Gap Metric and Fuzzy Model with Transfer Function
}

\author{
Gang $\mathrm{Li}^{1,2,3,4,5}$, Bin Liang ${ }^{1,2,3,4,5}$, Xueqian Wang ${ }^{1,2,3,4,5}$, Xiu Li $i^{1,2,3,4,5}$ and Rongjian Liang ${ }^{1,2,3,4,5}$ \\ ${ }^{1}$ Tsinghua University, National Laboratory for Information Science and Technology, 100084 Beijing, China \\ ${ }^{2}$ Tsinghua University, Graduate School at Shenzhen, 518055 Shenzhen, China \\ ${ }^{3}$ Tsinghua University, Department of Automation, 100084 Beijing, China \\ ${ }^{4}$ Shenzhen Key Lab of Space Robotic Technology and Telescience, 518055 Shenzhen, China \\ ${ }^{5}$ Shenzhen Engineering Lab of Precision Geometry Measurement Technology, 518055 Shenzhen, China
}

\begin{abstract}
This investigation is to deal with the modeling issue for nonlinear pressurized water reactor cores. Nonlinearity measure with gap metric and T-S fuzzy modeling are exploited to build the fuzzy model of a nonlinear core which approximates this nonlinear core model. The gap metric of the core is proposed to quantify the core nonlinearity. The curve in the whole range of core power level that is solved adopting the gap metric is the core nonlinearity measure. In terms of the measure, six linearized core models at six power levels are selected as local models of the nonlinear core model. Based on the local models and the introduction of the triangle membership function, the core fuzzy model is achieved. The core fuzzy model and nonlinear core model are simulated. Simulation results show that the core fuzzy model can approximate and substitute the nonlinear core model.
\end{abstract}

\section{Introduction}

Nuclear energy as a kind of clean energy provides an incentive to sustainable development of nuclear power plants (NPPs) for electricity generation. Meanwhile, of research fields for NNPs, the modeling study on plants of NPPs such as reactor cores is always popular. The pressurized water reactor (PWR) cores in NPPs possess nonlinear characteristics, and parameters of them are varying at different operating conditions. Hence, cores are essentially time-varying complex systems so that modeling their models is difficult. It is necessary and meaningful to research the modeling issue of nonlinear cores.

Many researchers have utilized the point reactor core modeling method to construct PWR core models at the full power level. Kerlin et al.[1] modelled the PWR core of the H. B. Robinson NPP at the full power level which was also verified to be correct. PWR cores at the full power level were modeled by Edwards et al.[2-5]to design core power control systems.

However, such modeling for cores based on one power level cannot represent the dynamics of nonlinear core within the global ranges of power level. Stimulated by the considerations, the work in this paper is to model a nonlinear PWR core within the global ranges of power level by the use of gap metric to quantify the core nonlinearity measure and the T-S fuzzy principle. Finally, the T-S fuzzy model and nonlinear model of the core are simulated, and conclusions are drawn.

\section{PWR core model}

The point reactor core modeling as a traditional modelling method is adopted to model a PWR core, which is a lumped parameter method. For this modeling, a core is regarded as one point without any space profile, and parameters of the core only vary with time and have nothing to do with space positions.

Table 1. Main model parameters.

\begin{tabular}{|c|c|}
\hline Parameter & Name \\
\hline$P_{r}$ & core power level \\
\hline$c_{r i}$ & $\begin{array}{c}\text { ith group normalized precursor } \\
\text { concentration }\end{array}$ \\
\hline$c_{r}$ & normalized precursor concentration \\
\hline$T_{f}$ & fuel average temperature \\
\hline$T_{i}$ & coolant inlet temperature \\
\hline$T_{e}$ & coolant outlet temperature \\
\hline$\delta r o d$ & $\begin{array}{c}\text { position variation of the control rod } \\
\text { (fraction of core length) }\end{array}$ \\
\hline$\delta$ & $\begin{array}{c}\text { deviation of a parameter from initial } \\
\text { steady-state value }\end{array}$ \\
\hline
\end{tabular}


According to the point reactor core modeling [1-3], the nonlinear PWR core is modelled adopting the point kinetics equations with six groups of delayed neutrons and reactivity feedbacks due to control rod movement and variations in fuel temperature and coolant temperature. Main model parameters are given in Table 1. The nonlinear core model is showed as Eqs.(1)-(6).

$$
\begin{aligned}
& \frac{d P_{r}}{d t}=\frac{\rho-\beta}{\Lambda} P_{r}+\sum_{i=1}^{g} \frac{\beta_{i} c_{r i}}{\Lambda} \\
& \frac{d c_{r i}}{d t}=\lambda_{i} n_{r}-\lambda_{i} c_{r i}, i=1,2, \ldots, g \\
& \frac{d T_{f}}{d t}=\frac{f_{f} P_{0}}{\mu_{f}} P_{r}-\frac{\Omega}{\mu_{f}} T_{f}+\frac{\Omega}{2 \mu_{f}} T_{i} \\
& +\frac{\Omega}{2 \mu_{f}} T_{e} \\
& \frac{d T_{e}}{d t}=\frac{\left(1-f_{f}\right) P_{0}}{\mu_{c}} n_{r}+\frac{\Omega}{\mu_{c}} T_{f}+\frac{2 M-\Omega}{2 \mu_{c}} T_{i} \\
& -\frac{2 M+\Omega}{2 \mu_{c}} T_{e} \\
& \rho=\rho_{r o d}+\alpha_{f}\left(T_{f}-T_{f 0}\right)+\frac{\alpha_{c}}{2}\left(T_{i}-T_{i 0}\right) \\
& +\frac{\alpha_{c}}{2}\left(T_{e}-T_{e 0}\right) \\
& \delta \rho_{\text {rod }}=\alpha_{\text {rod }} \delta \text { rod }
\end{aligned}
$$

The small perturbation linearization methodology is utilized to linearize this nonlinear core model, and the linearized core model is expressed by Eqs.(7)-(11).

$$
\begin{gathered}
\frac{d \delta P_{r}}{d t}=-\frac{\beta}{\Lambda} \delta P_{r}+\sum_{i=1}^{g} \frac{\beta_{i}}{\Lambda} \delta c_{r_{i}}+\frac{P_{r 0}}{\Lambda} \delta \rho \\
\frac{d \delta c_{r i}}{d t}=\lambda_{i} \delta n_{r}-\lambda_{i} \delta c_{r i}, i=1, \ldots, g \\
\frac{d \delta T_{f}}{d t}=\frac{f_{f} P_{0}}{\mu_{f}} \delta n_{r}-\frac{\Omega}{\mu_{f}} \delta T_{f}+\frac{\Omega}{2 \mu_{f}} \delta T_{i}
\end{gathered}
$$

$$
\begin{aligned}
\frac{d \delta T_{e}}{d t}= & \frac{\left(1-f_{f}\right) P_{0}}{\mu_{c}} \delta n_{r}+\frac{\Omega}{\mu_{c}} \delta T_{f} \\
& +\frac{2 M-\Omega}{2 \mu_{c}} \delta T_{i}-\frac{2 M+\Omega}{2 \mu_{c}} \delta T_{e} \\
\delta \rho= & \alpha_{\text {rod }} \delta \operatorname{rod}+\alpha_{f} \delta T_{f}+\frac{\alpha_{c}}{2} \delta T_{i} \\
& +\frac{\alpha_{c}}{2} \delta T_{e}
\end{aligned}
$$

One group delayed neutron model is utilized and the coolant inlet temperature is treated as a constant [2-4]. The transfer function and the state equation of the core are separately calculated and expressed by

$$
\begin{gathered}
G=\frac{\delta P_{r}}{\delta \operatorname{rod}}=\frac{\sum_{i=0}^{3} a_{i} s^{i}}{\sum_{i=0}^{4} b_{i} s^{i}} \\
\left\{\begin{array}{l}
\dot{\mathbf{x}}=A \mathbf{x}+B u \\
y=C \mathbf{x}+D u
\end{array}\right.
\end{gathered}
$$

where $u=\delta r o d$-the input; $y=\delta P_{r}$-the output; $a_{i}(i=0,1,2,3)$ numerator coefficients; $\quad b_{i} \quad(i=0,1,2,3,4)$-denominator coefficients; $s$-laplace operator; $\mathbf{x}=\left[x_{1}, x_{2}, x_{3}, x_{4}\right]^{\mathrm{T}}=\left[\delta P_{r}\right.$, $\left.\delta c_{r}, \delta T_{f}, \delta T_{e}\right]^{\mathrm{T}}$-the state matrix; $A$-the $\mathrm{R}^{4 \times 4}$ system matrix; $B$-the $\mathrm{R}^{4 \times 1}$ input matrix; $C$-the $\mathrm{R}^{1 \times 4}$ output matrix; $D$-the zero matrix.

A transfer function of the core at a power level $10 * i \%$ is denoted by $G_{10^{*} i \%}(i=1, \ldots, 10)$. These transfer functions are calculated by using parameters from Ref. [6], in which the total primary heat output is $2200 \mathrm{MW}$, the primary coolant inlet temperature is $285^{\circ} \mathrm{C}$, the primary coolant outlet temperature is $317^{\circ} \mathrm{C}$, the primary coolant average pressure is $15.5 \mathrm{MPa}$, and the primary coolant mass flow is $12861.1 \mathrm{~kg} / \mathrm{s}$.

\section{Nonlinearity measure with gap metric for core}

The nonlinearity measure on the basis of the gap metric for the core is proposed to quantify the core nonlinearity. One group of linearized core models can be selected to approximate the nonlinear core in terms of distributing situations of the core nonlinearity measure in the while range of power level, and each linear model is regarded as a local model of the core.

According to the work $[7,8]$, the gap metric of the core is developed as follows to solve the core nonlinearity. 
Let $O_{i}(i=1,2)$ be two closed linear operators in a Hilbert space $\mathrm{H}_{\mathrm{s}} . \mathrm{H}_{\mathrm{s}} \times \mathrm{H}_{\mathrm{s}}$ is also a Hilbert space with inner product derived from $\mathrm{H}_{\mathrm{s}}$. The graph of $O_{i}$ is denoted by $g_{r}\left(O_{i}\right)$. As $O_{i}$ is linear, $g_{r}\left(O_{i}\right)$ is a subspace of the product Hilbert space $\mathrm{H}_{\mathrm{s}} \times \mathrm{H}_{\mathrm{s}}$.

Definition 3.1. The gap between two closed linear operators $O_{1}$ and $O_{2}$ is defined by the gap between $g_{r}\left(O_{1}\right)$ and $g_{r}\left(O_{2}\right)$ :

$$
\Delta\left(O_{1}, O_{2}\right)=\Delta\left(g_{r}\left(O_{1}\right), g_{r}\left(O_{2}\right)\right)
$$

where $\Delta(\cdot)$ is a metric.

Linear systems such as $G$ of the core are viewed as mappings in a Hilbert space. Hence, $G$ is treated as an operator. [7].

Lemma 3.1. Frequency response systems are closed

According to Lemma 3.1, $G$ as a frequency response system is a closed linear operator in $\mathrm{H}_{\mathrm{s}}$. The gap metric in Definition 3.1 can be used to qualify the gap between two linearized systems of the core. So Eq.(15) is obtained by

$$
\Delta\left(G_{10^{*} i \%}, G_{10^{*} j \%}\right)=\Delta\left(g_{r}\left(G_{10^{*} i \%}\right), g_{r}\left(G_{10^{*} j \%}\right)\right)
$$

where $\Delta(\cdot) \in\left[\begin{array}{ll}0 & 1\end{array}\right] ; i \neq j ; i, j=1, \ldots, 10$.

Let $\mathrm{H}_{\infty}$ and $\mathrm{H}_{2}$ be the standard Hardy spaces of functions, and let $\mathrm{RH}_{\infty}$ be the subspace of real rational functions in $\mathrm{H}_{\infty} . \mathrm{G}_{10 * i \%}$ is a $1 \times 1$ rational transfer matrix and has the normalized right coprime factorization as follows.

$$
G_{10^{*} i \%}=N_{1} M_{1}^{-1}, G_{10^{*} j \%}=N_{2} M_{2}^{-1}
$$

with $M_{1}{ }^{*} M_{1}+N_{1}{ }^{*} N_{1}=\mathrm{I}$ and $M_{2}{ }^{*} M_{2}+N_{2}{ }^{*} N_{2}=\mathrm{I}$. where for $i=1$ or $2, M_{i}$ and $N_{i}$ belong to $\mathrm{RH}_{\infty}, M_{i}(\mathrm{~s})^{*}=M_{i}(-$ s) ${ }^{\mathrm{T}}, N_{i}(\mathrm{~s})^{*}=N_{i}(-\mathrm{s})^{\mathrm{T}}$.

$g_{r}\left(G_{10 * i \%}\right)$ or $g_{r}\left(G_{10 * j \%}\right)$ is a closed subspace of $\mathrm{H}_{2} \times \mathrm{H}_{2}$ that consists of all pairs $\left(\delta r o d, \delta P_{r}\right)$ such that $\delta P_{r}=G_{10 * i \%} * \delta \operatorname{rod}$ or $\delta P_{r}=G_{10 * j \%} * \delta \operatorname{rod}$. They are presented by

$$
g_{r}\left(G_{10^{*} i \%}\right)=\left[\begin{array}{c}
M_{1} \\
N_{1}
\end{array}\right] H_{2}, g_{r}\left(G_{10^{*} j \%}\right)=\left[\begin{array}{c}
M_{2} \\
N_{2}
\end{array}\right] H_{2}
$$

And

$$
\Delta\left(g_{r}\left(G_{10^{*} i \%}\right), g_{r}\left(G_{10^{*} j \%}\right)\right)=\left\|\Pi_{g_{r}\left(G_{\left.100^{*} \%_{0}\right)}\right.}-\Pi_{g_{r}\left(G_{10^{*} j \%}\right)}\right\|
$$

where $\prod_{()}$is the orthogonal projection onto $(\cdot)$.

Lemma 3.2. The gap between $G_{10^{*} i \%}$ and $G_{10 * j \%}$ is calculated by

$$
\begin{aligned}
& \Delta\left(G_{10^{*} i \%}, G_{10^{*} j \%}\right) \\
& =\left\|\Pi_{g_{r}\left(G_{\left.10 r^{*} \%_{0}\right)}\right.}-\Pi_{g_{r}\left(G_{\left.100^{*} \%_{0}\right)}\right.}\right\| \\
& =\max \left\{\vec{\Delta}\left(G_{10^{*} i \%}, G_{10^{*} j \%}\right), \vec{\Delta}\left(G_{10^{*} j \%}, G_{10 *_{i} \%}\right)\right\} \\
& =\max \left\{\inf _{q \in H_{\infty}}\left\|\left[\begin{array}{c}
M_{1} \\
N_{1}
\end{array}\right]-\left[\begin{array}{c}
M_{2} \\
N_{2}
\end{array}\right] q\right\|_{\infty}, \inf _{q \in H_{\infty}}\left\|\left[\begin{array}{c}
M_{2} \\
N_{2}
\end{array}\right]-\left[\begin{array}{c}
M_{1} \\
N_{1}
\end{array}\right] q\right\|_{\infty}\right\}
\end{aligned}
$$

where $\vec{\Delta}(\cdot)$ denotes a directed gap.

\section{Fuzzy modeling}

The T-S fuzzy method [9] can be used to handle modeling issues of nonlinear plants such as the core at varying operating conditions. According to this modeling, the power level operating range of the nonlinear core is partitioned into separate subregions, local dynamics in each region are represented by one linear model as a local model of this core; fuzzy rules are proposed adopting membership functions that are utilized to develop fuzzy sets of which the intersection of two ones is nonempty, the T-S fuzzy model that is the overall model to approximate the nonlinear core is then achieved via "blending" of all linear local models based on fuzzy rules.

\subsection{Core local models based on nonlinearity measure}

$G_{10 \%}$ is chosen as a referenced model and a gap metric between $G_{10 \%}$ and $G_{P r}$ for $P_{r} \in\left[\begin{array}{ll}0.1 & 1\end{array}\right]$ is calculated by means of Section 3. This calculated gap metric is the core nonlinearity measure between $10 \%$ and $P_{r}$.

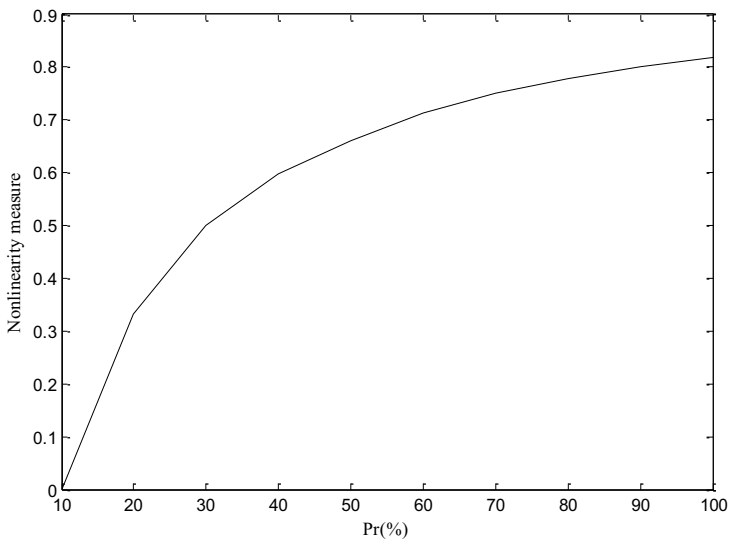

Figure 1. Nonlinearity measure of the core

The nonlinearity measure curves of the core are obtained and shown in Fig.1, where the curve approximates a parabola. Comparing with two level areas $\left[\begin{array}{ll}0.1 & 0.6\end{array}\right]$ and $\left(\begin{array}{ll}0.6 & 1.0\end{array}\right]$, the curve change relatively rapidly in $\left[\begin{array}{ll}0.1 & 0.6\end{array}\right]$, and vary relatively gently in $\left(\begin{array}{lll}0.6 & 1.0\end{array}\right]$ in the form of an approximated straight line. These indicate that the core has the strong nonlinearity in $\left[\begin{array}{ll}0.1 & 0.6\end{array}\right]$ and the weak nonlinearity in $\left(\begin{array}{ll}0.6 & 1.0\end{array}\right]$. Therefore, choose three linear models at $10 \%, 20 \%, 40 \%$ and $60 \%$ to substitute the nonlinear core in $\left[\begin{array}{ll}0.1 & 0.6\end{array}\right]$ with the strong nonlinearity, select two linear models at $80 \%$ and $100 \%$ to substitute the nonlinear core in $\left(\begin{array}{lll}0.6 & 1.0\end{array}\right]$ with the weak nonlinearity. Finally, linearized core models at five levels are selected as local models of the core to substitute the nonlinear core in $(0100 \%$ ].

Let $G_{1}=G_{10 \%}, G_{2}=G_{20 \%}, G_{3}=G_{40 \%}, G_{4}=G_{60 \%}, G_{5}=G_{80 \%}$, $G_{6}=G_{100 \%}$. The state equation of $G_{i}$ is represented by $\left(A_{i}\right.$ $\left.B_{i} C_{i} D_{i}\right)(i=1, \ldots, 6)$. Parameters of $G_{5}$ are enumerated: [ $a_{3}$, $\left.a_{2}, a_{1}, a_{0}\right]=\left[\begin{array}{llll}5088 & 7021 & 1853 & 137.2\end{array}\right],\left[b_{4}, b_{3}, b_{2}, b_{1}, b_{0}\right]=[1$ $401.4692 .2389 .943 .2] . G_{1}, G_{2}, G_{3}, G_{4}$ and $G_{5}$ are used to respectively denote dynamics of the nonlinear core located in the five power level ranges $(0,10 \%],[10 \%$, 
$20 \%],[20 \%, 40 \%],[40 \%, 60 \%],[60 \%, 80 \%]$ and $[80 \%$, $100 \%]$ in turn.

\subsection{Core fuzzy model}

The triangle membership function is utilized to set up fuzzy rules. The membership function of the core fuzzy model at whole power levels is shown in Fig.2. form.

The fuzzy logic system (FLC) is of the following

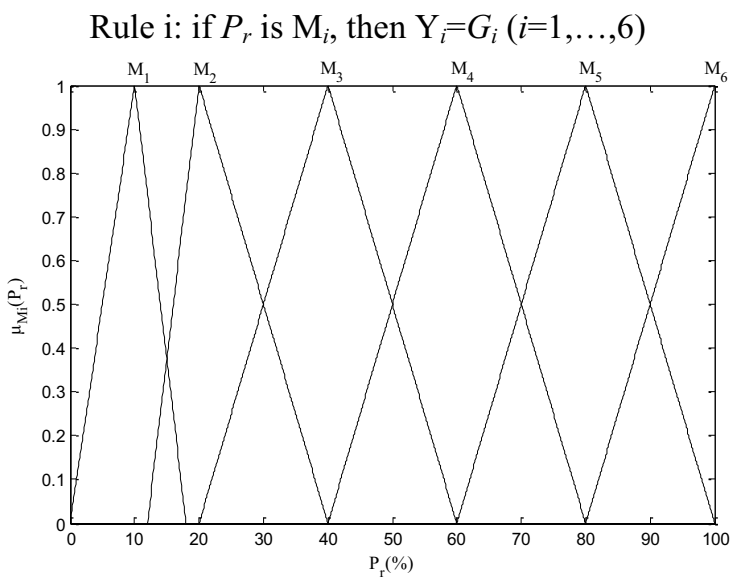

Figure 2. Membership function of core fuzzy model at whole power levels

where Rule $i$ denotes the $i$ th fuzzy rule; $\mathrm{M}_{1}, \mathrm{M}_{2}, \mathrm{M}_{3}, \mathrm{M}_{4}$, $\mathrm{M}_{5}$ and $\mathrm{M}_{6}$ respectively represent the fuzzy sets corresponding to the power levels $10 \%, 20 \%, 40 \%, 60 \%$, $80 \%$ and $100 \% ; \mu_{\mathrm{Mi}}\left(P_{r}\right)$ represents the membership when a power $P_{r}$ belongs to the fuzzy set $\mathrm{M}_{i}$, which is calculated in the light of a relationship between the membership and the core power level.

In the light of Fig.2, weight values $q_{i}$ for the "blending" of $G_{i}(i=1, \ldots, 6)$ is calculated as Eq. (20).

$$
q_{i}=\frac{\mu_{M_{i}}\left(P_{r}\right)}{\sum_{i=1}^{6} \mu_{M_{i}}\left(P_{r}\right)}, i=1, \ldots, 6
$$

where $P_{r} \in\left(\begin{array}{ll}0 & 1\end{array}\right], q_{i} \in\left[\begin{array}{ll}0 & 1\end{array}\right]$

One value $q_{i}$ indicates how the $i$ th local model at a power level belongs to a core fuzzy model at this power level. Finally, the core fuzzy model is expressed by Eq.(21) or (22).

$$
\begin{gathered}
G_{F M}=\sum_{i=1}^{6} q_{i} G_{i} \\
\left\{\begin{array}{l}
\dot{\mathbf{x}}=\sum_{i=1}^{6} q_{i}\left[A_{i} \mathbf{x}+B_{i} u\right] \\
y=\sum_{i=1}^{6} q_{i} C_{i} \mathbf{x}
\end{array}\right.
\end{gathered}
$$

\section{Simulation}

To verify effectiveness and correctness of this fuzzy modeling for the nonlinear core, the core fuzzy model (21) and the core nonlinear model are compared via simulations.

When the input $\delta$ rod is taken as a 0.01 step, the fuzzy modeland the nonlinear model at ten typical power levels $10 \times i \%(i=1, \ldots, 10)$ are simulated, and output responses of the fuzzy modeland nonlinear model at these levels are shown in Fig.3, which is namely responses of $\delta P_{r}$. In fig. 3 , the curve $\mathrm{FM} i(i=1, \ldots, 10)$ represents the output response of the fuzzy model at $10 \times i \%$, and the curve NL $i$ $(i=1, \ldots, 10)$ is the output response of the nonlinear model at $10 \times i \%$. From FM $i$ and $\mathrm{NL} i$, it can be observed that FM $i$ approaches NL $i$. Similarly, the similar simulation results are able to be achieved for other power levels.
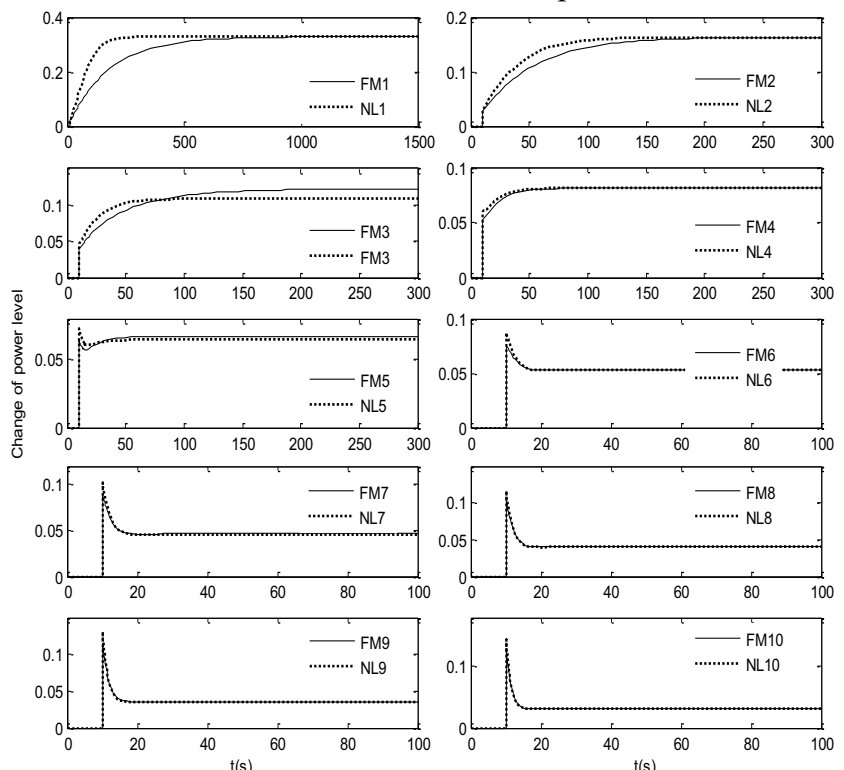

Figure 3. Output responses of fuzzy model and nonlinear model for the core at ten power levels

Hence, the core fuzzy model can approximate the core nonlinear model according to simulation results.

\section{Conclusions}

The work handles the modeling problem of nonlinear PWR cores. On the basis of modeling a nonlinear core and calculating the core nonlinear measure with the introduction of the gap metric, one group linear local models of the core is defined. In terms of local models and the triangle membership function, the T-S fuzzy model for the core is developed. From numerical simulations, it is obtained that the core fuzzy model can substitute the core nonlinear model. The proposed modeling principle as a reference method can be applied to model other reactor cores and nonlinear plants, and also applicable for stability analysis and control system design of nonlinear systems.

\section{Acknowledgments}

The authors would like to thank anonymous reviewers for their valuable comments. The work is funded by National High Technology Research and Development Program of 
China (863 Program) (No.2015AAXX46611) and China

Postdoctoral Science Foundation (No.20159200078).

\section{References}

1. T.W. Kerlin, E.M. Katz, J.G. Thakkar, J.E. Strange, Theoretical and experimental dynamic analysis of the H. B. Robinson nuclear plant, Nuclear Technology, 30, 299-316 (1976).

2. R.M. Edwards, K.Y. Lee, M.A. Schultz, State feedback assisted classical control: an incremental approach to control modernization of existing and future nuclear reactors and power plants, Nuclear Technology, 92, 167-185 (1990).

3. A. Ben-Abdennour, R.M. Edwards, K.Y. Lee, LQG/LTR robust control of nuclear reactors with improved temperature performance, IEEE Trans. on Nuclear Science, 39, 2286-2294 (1992).

4. H. Arab-Alibeik, S. Setayeshi, Improved temperature control of a PWR nuclear reactor using an LQG/LTR based controller, IEEE Trans. on Nuclear Science, 50, 211-218 (2003).

5. G.R. Ansarifar, H.R. Akhavan, Sliding mode control design for a PWR nuclear reactor using sliding mode observer during load following operation, Annals of Nuclear Energy, 75, 611-619 (2015).

6. IAEA, Directory of Nuclear Reactors Vol.IX, 27-34, (International Atomic Energy Agency, 1971).

7. A.K. El-Sakkary, The gap metric: robustness of stabilization of feedback systems, IEEE Trans. Autom. Control, AC-30, 240 (1985).

8. T.T. Georgiou, On the computation of the gap metric, Proc. Of 27th Conference on Decision and Control, 1360 (1988).

9. K. Tanaka, M. Sugeno, Stability analysis and design of fuzzy control systems, Fuzzy Sets and Systems, 45, 135-156 (1992). 